\title{
Fulminant Puerperal Sepsis caused by Hemolytic Group A Streptococci and Toxic Shock Syndrome - A Case Report and Review of the Literature
}

\author{
Foudroyante Puerperalsepsis durch hämolysierende Streptokokken Gruppe A \\ mit Toxic-Shock-Syndrom - Fallbericht und Literaturübersicht
}

Authors

Affiliations
G. J. Bauerschmitz ${ }^{1}$, M. Hellriegel ${ }^{1}$, J. Strauchmannn ${ }^{2}$, J. Schäper ${ }^{2}$, G. Emons ${ }^{1}$

${ }^{1}$ Frauenklinik, UMG, Göttingen

2 Zentrum für Anästhesie, UMG, Göttingen
Deutschsprachige Zusatzinformationen online abrufbar unter: www.thieme-connect.de/ ejournals/toc/gebfra

$\begin{array}{ll}\text { received } & 6.11 .2013 \\ \text { revised } & 12.5 .2014 \\ \text { accepted } & 24.6 .2014\end{array}$

Bibliography

DOI http://dx.doi.org/

10.1055/s-0034-1382854

Geburtsh Frauenheilk 2014; 74 :

764-767 @ Georg Thieme

Verlag KG Stuttgart . New York. ISSN 0016-5751

\section{Correspondence}

PD Dr. Gerd J. Bauerschmitz

UMG

Frauenklinik

Robert-Koch-Straße 40

37075 Göttingen

gerd.bauerschmitz@

med.uni-goettingen.de

\section{Introduction}

$\nabla$

Most physicians would consider puerperal sepsis, known colloquially as childbed fever, to be a relic of the past which has long since been overcome with the introduction of Semmelweis' hygiene measures in general practice. Puerperal fever does not feature in the ordinary experience of most people.

But puerperal sepsis caused by Group A Streptococci (Streptococcus pyogenes) may take a highly dramatic course and can result in lifelong damage [1]. There are regular reports of such cases in the literature [2-5].

We present a case of puerperal sepsis which occurred unusually early, describe its severe course and discuss the case in the light of the recent literature.

\section{Case Report}

A 42-year-old gravida III/para II was admitted to hospital in the $28+1$ week of gestation for uncontrolled pre-existing arterial hypertension (BP 200/110) and suspicion of placental insufficiency on Doppler sonography. There were no indications of superimposed preeclampsia, and laboratory and urine parameters were within normal ranges. Fetal growth and amount of amniotic fluid were in the lower range of standard values, and the values for umbilical resistance and bilateral uterine flow were borderline pathological. Vaginal pH was 4; Pap smear and CTG done on admission were unremarkable. Subjectively, the patient had no symptoms. Apart from the hypertension, the patient's medical history was unremarkable. Betamethasone was administered $(2 \times 12 \mathrm{mg}$ at an interval of 24 hours) to induce fetal lung maturation, and drugs were administered in an attempt to reduce blood pressure while shielding the patient. Doppler sonography showed a dra- matic worsening of placental insufficiency after the administration of drugs for lung maturation, with zero umbilical flow and intermittent reverse flow. When CTG became suspicious, the infant was delivered by cesarean section under spinal anesthesia. Except for a large myoma on the anterior wall which displaced the uterine cavity and which had led to delayed development of the infant, findings were unremarkable and the amniotic fluid was clear. Single dose antibiotic prophylaxis (cefazolin $2 \mathrm{~g}$ ) was administered intravenously after the infant was delivered. The neonate weighed $980 \mathrm{~g}$ (Apgar score: 1/6/8; pH: 7.35) and was transferred to the premature baby unit for further care.

Initially, the postoperative course was entirely unremarkable. But around 16 hours later, the patient developed acute shock. She presented with pronounced systemic inflammatory response syndrome (SIRS) with shortness of breath, a drop of oxygen saturation to $80 \%$ in room air, and tachycardia with a mean systolic pressure of 130 and a BP of $80 / 40$ but without strong pain, fever or clearly attributable laboratory findings. The only remarkable finding was leukopenia of 2800/ $\mu \mathrm{l}$; the significantly increased CRP (C-reactive protein) level of $22.7 \mathrm{mg} / \mathrm{l}$ was considered to be a postoperative surge. The patient's respiratory frequency and temperature were within normal ranges. Thoracic CT performed to exclude pulmonary embolism was unremarkable. Abdominal sonographies carried out to exclude secondary hemorrhage showed increasing amounts of free fluid in the abdomen. The patient's clinical condition worsened alarmingly, and re-laparotomy was done because of a suspicion of secondary hemorrhage. Two liters of putrid fluid were found in the abdomen. The uterus was soft, and the scar of the cesarean section was clinically intact. A diagnosis of puerperal sepsis was made; a hysterectomy procedure with lavage was carried out; the retroperitoneal space was opened with revision 
surgery of the ovarian vessels up to the vena cava, aorta and renal vein to exclude septic thrombus. Revision surgery of the intestines was done in collaboration with colleagues from the Department of Abdominal Surgery (no pathological findings) with extensive abdominal lavage and the placement of large abdominal drains. The patient was transferred to the intensive care unit in a moderately stable hemodynamic condition, and anticoagulation therapy was initiated.

The patient required high doses of catecholamines and presented with severe oxygenation dysfunction with a $\mathrm{paO}_{2}$ of $100 \mathrm{mmHg}$ at $100 \%$ oxygen (oxygenation index 100 ). Because of the severity of symptoms, antibiotic therapy (which had commenced immediately preoperatively) was switched from cefazolin and metronidazole to meropenem as empiric therapy. CRP was now $142 \mathrm{mg} / \mathrm{l}$. The laboratory findings of the swab obtained intraoperatively were not yet available at this point.

As the noradrenalin doses were increased, a PICCO catheter (for pulse contour cardiac output measurement) was inserted for close hemodynamic monitoring. Cardiac ultrasound was done and demonstrated a good pump function of the left heart. Volume loading did little to decrease the high lactate levels. Oxygenation improved very gradually. Acute renal failure additionally set in after revision surgery, requiring continuous hemodiafiltration (CVVHDF) to treat early hyperkalemia. The patient received coagulation factors (PPSB $=$ prothrombin complex concentrate) and thrombocyte substitution with ROTEM ${ }^{\circledR}$-assisted monitoring (rotational thrombo-elastometry to measure the full blood coagulation profile) because of coagulation failure and clinical bleeding tendency.

At this point, incipient hypoglycemia developed, indicating impaired hepatic synthesis, and substitution was required. Repeat abdominal ultrasound showed no change in findings.

Because of the patient's poor hemodynamic condition, the patient was sedated with midazolam and ketamine (Richmond Agitation-Sedation Scale [RASS]: minus 4).

The patient continued to require high doses of noradrenalin. Antibiotic therapy was expanded on the 2nd postoperative day to include linezolid because of the limited effectiveness of meropenem against Gram-positive cocci and the continued rise of CRP to $182 \mathrm{mg} / \mathrm{l}$. Therapeutic anticoagulation values were achieved. The patient developed therapy-refractory lactate acidosis which could not be remedied by CVVHDF or by the continuous administration of TRIS buffer solution (Tris[hydroxymethyl] aminomethane hydrochloride to treat metabolic acidosis).

Because of the high catecholamine doses which indicated catecholamine-resistant shock, methylene blue was administered intermittently to reduce NO-induced relaxation of the smooth vascular muscles. Ongoing coagulation failure required continuous substitution with thrombocyte concentrate. Heparin/platelet factor 4 antibody test was done to exclude heparin-induced thrombocytopenia type II. There were no bleeding stigmata.

Streptococcus pyogenes was detected on the abdominal swab obtained intraoperatively during re-laparotomy. The microbes were also detected in the vaginal smear obtained during the same operation.

Histological analysis was unable to find the foci. The uterine scar was adequate with no evidence of inflammation. The myoma was histologically unremarkable. Nevertheless, the presumption remains that the cause was ascending infection caused by Streptococcus pyogenes, although the origin of the Streptococci remains unclear, given the unremarkable familial history of diseases caused by Streptococci such as angina or scarlet fever. Pathologi- cal investigation only found extensive fibrinous peritonitis with adipose tissue necrosis.

The initially high levels of markers for infectious disease gradually dropped with anti-sepsis therapy, and anti-microbial therapy was de-escalated to ampicillin/sulbactam.

Catecholamine administration was discontinued on the 9th postoperative day, and the patient's pulmonary and metabolic condition continued to stabilize. The patient was put on parenteral nutrition.

After being administered antagonist drugs to reverse sedation, the patient opened her eyes but was not yet fully conscious at that point. CT scan of the skull showed no significant intracranial pathological findings. CT of the thorax and abdomen were also done at the same time. In addition to pleural effusions, the images showed bilateral hypodense hepatic lesions as well as thrombosis of the right external iliac vein. Findings were also suspicious for diffuse pulmonary infiltrates, compatible with scattered septic foci.

Further revision surgery of the abdominal wall was done on the 12th postoperative day because of pronounced impaired wound healing, which presented as necrotizing fasciitis on histological examination. Extensive wound debridement was done with subsequent placement of a VAC (Vacuum-Assisted Closure) system. No evidence of bacteria could be found. After revision surgery, the patient again required high doses of catecholamines. The continued absence of diuresis meant that CVVHDF also had to be continued. When the patient again presented with fever, leukocytosis and increased procalcitonin values, antibiotic therapy was switched to meropenem and linezolid. At the same time all central venous catheters including all dialysis catheters were changed. The exchange proved to be difficult because of thrombi in both internal jugular veins.

Revision surgery of the abdominal wall was done repeatedly, and the fascia could gradually be closed, although VAC therapy was continued. The patient developed wet gangrene of the 2nd toe on her left foot in consequence of the lengthy catecholamine administration, and the toe had to be amputated.

After the antibiotic regimen was changed on the 12th day the patient's hemodynamic condition continued to improve. Hemodiafiltration finally achieved negative equilibrium. The patient developed critical illness polyneuropathy/polymyopathy, and on the 18th day in the intensive care unit a tracheotomy was performed to help with weaning from mechanical ventilation. The abdominal wall was closed on the same day.

Feeding was changed from parenteral nutrition to enteral tube feeding through a stomach tube. But despite the assistance of prokinetic drugs, the passage of nutrients through the gastrointestinal tract continued to be difficult. The enteral load was gradually increased until nutrition was adequate.

Weaning off mechanical ventilation was protracted because of critical illness polyneuropathy/polymyopathy. After the patient's hemodynamics had stabilized and there were no more signs of infection, antibiotic therapy was de-escalated again after the 19th postoperative day and finally terminated. The patient now received intermittent dialysis via a single-lumen (Demers) catheter. However anticoagulation therapy was continued with sustained administration of up to 35000 IE/d heparin. Further revision surgeries of the abdominal wall followed by VAC treatment for impaired wound healing were required.

After weaning from mechanical ventilation the patient began her first attempts at swallowing and speaking. The symptoms of crit- 
ical illness polyneuropathy/polymyopathy decreased and the patient was mobilized.

After spending almost 2 months in intensive care, the patient was transferred to a surgical intermediate care station for further surgical care of the abdominal wound. By this time there were no indications of any neurological deficits, and the patient was mobile and stable. However, the patient had not yet returned to full oral feeding and still required dialysis.

A further 2 weeks on a general ward followed, during which the patient was able return to normal nutrition and was fully mobilized. Dialysis was discontinued during the course of one week after retention values had normalized. The patient was discharged home in an acceptable condition and received care and assistance from a mobile nursing service at home.

The patient continued to recover at home and has since recovered completely, both physically and psychologically, at 6 months postpartum.

\section{Discussion}

$\nabla$

There is currently no reliable data on the incidence of puerperal sepsis, even though all deaths in Germany from puerperal sepsis must be registered. Reports range from 0.2 to 1.0 deaths for every 100000 births $[1,2,6,7]$. Compared with a death rate of up to $20 \%$ of women who gave birth in the pre-Semmelweis era, this appears very low; however, it is estimated that a significant number of cases go unreported [8].

Puerperal sepsis is the most serious form of puerperal fever, which is defined as an infection affecting women following childbirth or a miscarriage. It is associated with a mortality of up to $50 \%$. The center of infection is usually the uterus with its large open wound after discharge of the placenta, which represents a portal of entry for microorganisms even under the most hygienic conditions. Physiologically, ascending bacteria are removed by the lochia, but decreased or retained lochia increases the risk of infection. The most common infectious microorganisms are Escherichia coli, Staphylococci and Streptococci [7-10]. The risk of infection is significantly increased after cesarean section compared to vaginal delivery $[11,12]$. The pathophysiological cause of this is unclear but it could be due to the decreased amount of lochia after cesarean section.

Typical early signs of puerperal sepsis, particularly if the infection is caused by Group A Streptococci, differ from the early signs of other infectious diseases. The infection therefore often goes unrecognized, and crucial antibiotic treatment is often started too late. Patients often only have a slight increase in temperature; severe cases in particular may often have no increase in temperature at all $[4,5,8]$. The main symptoms of puerperal sepsis are abdominal pain and a generalized feeling of fatigue. Laboratory values, particularly complete blood count without differential, may remain unremarkable for a long time [8]. This makes early diagnosis extremely difficult, and diagnosis is often only made when the patient has developed full blown sepsis, usually with pronounced respiratory insufficiency. Actual sepsis is relatively rare, as antibiotic therapy is often initiated ex juvantibus to treat patients with persistent unspecific symptoms, and the majority of patients are young and fully immunocompetent. But the mortality associated with advanced stage sepsis is enormously high with reported rates of $20-60 \%[3-5,8,13,14]$.

Group A Streptococci are very common bacteria which have a tendency to colonize the throat of around $20 \%$ of the population in the winter months without giving rise to symptoms. Transmission occurs by droplet and smear infection. In the majority of cases, Group A Streptococci originate from the vaginal area, which was colonized by the microorganisms prior to the birth. The bacteria enter the body through the endometrium or a vaginal injury, where they find ideal growth and propagation conditions. In addition to the rapid and systemic spread of the bacteria from the portal of entry throughout the whole body, the bacteria also release toxins which attack cells directly. Mortality from Group A streptococcal sepsis is precipitated by impaired pulmonary function and coagulation $[4,8]$.

So-called "toxic shock syndrome" represents a particular complication of Group A streptococcal sepsis [15-18]. Toxic shock syndrome can progress to multiple organ failure due to the potential for arterial leakage and tissue necrosis. Antibiotic therapy is unsuccessful to treat such cases as symptoms are no longer caused by the bacteria themselves but by the toxins released in large amounts by the bacteria [19]. The toxins cause a cytokine cascade with subsequent activation of neutrophils and mediators, leading to respiratory failure, excessive vascular permeability and shock; mortality is more than $50 \%$, even under the most modern medical conditions [16-18].

The diagnosis of puerperal sepsis is based on direct clinical examination of the patient. Cardinal symptoms include severe, generalized malaise, with or often without fever, as well as increased levels of the inflammatory marker CRP, results of a hemogram may initially be unremarkable. But there is a large gray area between a normal postpartum/postoperative course, local infection due to abscess, and puerperal sepsis, and experience is needed to differentiate between them in the early stages. It is often not possible to determine the specific pathogen, as smears or blood cultures are often only done after the patient has received initial doses of antibiotics. What is usually found are the resistant colonizing bacteria [8]. Nevertheless, it is important to continue searching for the pathogen by means of blood cultures and smear tests. In patients with sepsis the search for the pathogen should be an essential part of diagnosis and must start prior to the administration of antibiotics.

The therapy of choice for streptococcal infections is high doses of penicillin $\mathrm{G}$, as resistance to penicillin $\mathrm{G}$ has not been reported [4, 8]. If determination of the pathogen is not possible, the clinical symptoms should determine the choice of treatment, which should preferably take the form of a broad spectrum combination therapy $[4,8,20]$. Empirically, the therapy of choice consists of a combination of piperacillin with a $\beta$-lactamase inhibitor, or alternatively meropenem [20]. The importance of a hysterectomy in patients with puerperal sepsis is largely ignored in the German specialist literature, and in the textbooks it is generally considered as a last resort. There are no studies or Cochrane analyses showing the benefits of this approach. But in the current international literature, hysterectomy is considered an essential part of the surgical removal of septic foci in patients who do not respond immediately to adequate antibiotic treatment [21-22]. As Group A Streptococci rapidly attack all organs, in theory there is no single focus of septic infection any more. But the pathogen is never detected in time, meaning that surgical revision may be the only potentially life-saving approach that remains if conservative therapy fails. In a patient with manifest sepsis which does not respond to antibiotic treatment within a few hours, we are of the opinion that surgical revision must be done soon and must include the removal of all potential septic foci, followed by extensive lavage. This approach is indicated even if the pathogen has 
not (yet) been determined and is often carried out [9,21,22]. Hysterectomy removes a potential focus of septic infection. Clinically, uterine subinvolution may also be present which can encourage infection. Patients require careful examination to detect or exclude septic thrombus and loop abscess. Lavage of the abdomen reduces the bacterial and toxin load. Sepsis and ovarian vein thrombosis, which are otherwise only found on autopsy, can be detected in time and treated. Consequently, surgical revision continues to be beneficial and can be life-saving.

\section{Summary}

Puerperal sepsis is a rare but serious and potentially lethal syndrome. It is imperative that severe postpartum malaise is taken seriously; early initiation of antibiotic therapy before sepsis becomes manifest can save lives.

\section{Conflict of Interest}

\section{$\nabla$}

None.

\section{References:}

1 Peterson EE. Infektionen in Gynäkologie und Geburtshilfe: Lehrbuch und Atlas. 5. Auflage. Stuttgart: Georg Thieme Verlag KG; 2011

2 Hamilton SM, Stevens DL, Bryant AE. Pregnancy-related group A streptococcal infections: temporal relationships between bacterial acquisition, infection onset, clinical findings, and outcome. Clin Infect Dis 2013; 57: 870-876

3 Lapinsky SE. Obstetric infections. Crit Care Clin 2013; 29: 509-520

4 Rimawi BH, Soper DE, Eschenbach DA. Group A streptococcal infections in obstetrics and gynecology. Clin Obstet Gynecol 2012; 55: 864-874

5 Soper $D$. Early recognition of serious infections in obstetrics and gynecology. Clin Obstet Gynecol 2012; 55: 858-863

6 Schuitemaker N, van Roosmalen J, Dekker G et al. Increased maternal mortality in The Netherlands from group A streptococcal infections. Eur J Obstet Gynecol Reprod Biol 1998; 76: 61-64
7 Welsch H. Müttersterblichkeit während Geburt und Wochenbett bei vaginaler Entbindung und Sectio caesarea. Gynäkologe 1997; 30: 742-756

8 Petersen EE. Streptokokken-A-Puerperalsepsis. Gynäkologe 1999; 32: 512-517

9 Rothe K, Schrey S, Laudi $S$ et al. Fulminante Puerperalsepsis durch Streptokokken der Gruppe A. Geburtsh Frauenheilk 2013; 73: P4_6

10 Gramm S, Hiden M, Kraschl J et al. Postpartales Staphylococcus aureus induziertes Toxic Shock Syndrom (STSS). Geburtsh Frauenheilk 2009; 69: P15

11 Burrows LJ, Meyn LA, Weber AM. Maternal morbidity associated with vaginal versus cesarean delivery. Obstet Gynecol 2004; 103 (5 Pt 1): 907-912

12 Declercq E, Barger M, Cabral HJ et al. Maternal outcomes associated with planned primary cesarean births compared with planned vaginal births. Obstet Gynecol 2007; 109: 669-677

13 Hackett SP, Stevens DL. Streptococcal toxic shock syndrome: synthesis of tumor necrosis factor and interleukin- 1 by monocytes stimulated with pyrogenic exotoxin A and streptolysin O. J Infect Dis 1992; 165: 879-885

14 Jorup-Ronstrom C, Hofling M, Lundberg Cet al. Streptococcal toxic shock syndrome in a postpartum woman. Case report and review of the literature. Infection 1996; 24: 164-167

15 Al-ajmi JA, Hill P, O'Boyle C et al. Group A Streptococcus toxic shock syndrome: an outbreak report and review of the literature. J Infect Public Health 2012; 5: 388-393

16 Bruun T, Kittang BR, de Hoog BJ et al. Necrotizing soft tissue infections caused by Streptococcus pyogenes and Streptococcus dysgalactiae subsp. equisimilis of groups $C$ and $G$ in western Norway. Clin Microbiol Infect 2013; 19: E545-E550

17 Kimber I, Nookala S, Davis CC et al. Toxic shock syndrome: characterization of human immune responses to TSST-1 and evidence for sensitivity thresholds. Toxicol Sci 2013; 134: 49-63

18 Low DE. Toxic shock syndrome: major advances in pathogenesis, but not treatment. Crit Care Clin 2013; 29: 651-675

19 Mason KL, Aronoff DM. Postpartum group A Streptococcus sepsis and maternal immunology. Am J Reprod Immunol 2012; 67: 91-100

20 Institut für Medizinische Mikrobiologie und Krankenhaushygiene der HHU. Leitfaden Antiinfektiva und Infektionserkrankungen - Praktische Hinweise zur antimikrobiellen Therapie. 2. Auflage. Düsseldorf: Heinrich-Heine-Universität; 2009

21 Soper $D E$. Early recognition of serious infections in obstetrics and gynecology. Clin Obstet Gynecol 2012; 55: 858-863

22 Rimawi BH, Soper DE, Eschenbach DA. Group A streptococcal infections in obstetrics and gynecology. Clin Obstet Gynecol 2012; 55: 864-874 